\title{
Desarrollo de capacidades para la operación del reactor nuclear IAN-R1
}

\section{Development of the capacities for the operation of the nuclear reactor IAN-R1}

\author{
Jaime Sandoval Lagos ${ }^{1}$, Edgar Mauricio López ${ }^{1}$
}

Citación: J. Sandoval Lagos y E. M. López, “Desarrollo de capacidades para la operación del reactor nuclear IAN-R1”, Revista de Investigaciones y Aplicaciones Nucleares n. ㄹ 2, pp. 15-30, 2018.

Recibido: 20 de junio de 2018

Aceptado: 9 de noviembre de 2018

Publicado en línea: 17 de

diciembre de 2018

Doi: $10.32685 / 2590-7468 /$ invapnuclear.2.2018.52
Palabras clave: IAN-R1, reactor

nuclear de investigación, utilización del reactor, análisis por activación neutrónica, irradiación.

\section{RESUMEN}

Este documento presenta una descripción histórica del reactor nuclear de investigación IAN-R1. Se hace una contextualización sobre el origen del reactor en el marco del programa Átomos para la Paz, y se incluyen las características técnicas y la configuración inicial del núcleo, cuyo combustible nuclear de tecnología MTR (90\%) fue sustituido por uno de tipo TRIGA (20\%) (acrónimos de material testing reactor y training, research, isotopes general atomics, respectivamente).

De igual manera, se presentan las características de las dos modernizaciones que se han realizado a la instrumentación y el control. La primera está orientada a la instalación de tres canales nucleares - dos de amplio rango y un canal de potencia-, renovación de la consola de control y la instalación del gabinete del sistema de adquisición de datos (DAC); la segunda, correspondiente a la nueva instrumentación y el control del reactor, está orientada al cambio de la consola de control, que soporta los servidores de control y supervisión, un canal nuclear NP-1000, impresora, cuatro pantallas de la interface humano-máquina HMI, teclado del sistema de manejo de barras y dos teclados para cada uno de los servidores. Además, se realizó la sustitución del DAC por el gabinete de la instrumentación, del cual forman parte los sistemas de protección del reactor, sistema redundante de control y el sistema de supervisión. La instrumentación y control se caracteriza por el empleo del estándar Ethernet para lograr la interconectividad de los sistemas, programación de la interface humano-máquina (HMI) empleando código de fuente abierta Java ${ }^{\mathrm{tm}}$, y multiplataforma, separación lógica de funciones aplicando conceptos de control distribuido y modularidad, redundancia, criterio de falla única e independencia.

Se muestra la utilización del reactor refiriendo las facilidades de irradiación disponibles para irradiación de materiales para ser estudiados mediante la técnica de análisis por activación neutrónica (AAN). Del mismo modo, está prevista la irradiación en apoyo de la utilización de la técnica de datación por huellas de fisión, la investigación y el apoyo a instituciones educativas mediante conferencias técnicas y visita a la instalación nuclear.

${ }^{1}$ Servicio Geológico Colombiano, Dirección de Asuntos Nucleares

Email de correspondencia: jsandova@sgc.gov.co 
Keywords: IAN-R1, nuclear research reactor, reactor utilization, neutron activation analysis, irradiation.

\section{ABSTRACT}

This document presents a historical description of the nuclear research reactor IAN-R1. A contextualization is made about the origin of the reactor within the framework of the Atoms for Peace program, including the technical characteristics and the initial configuration of the core, which was replaced by nuclear fuel MTR technology (90\%) to a new fuel type TRIGA (20\%) (acronyms of material testing reactor and training, research, isotopes, general atomics, respectively.

In the same way, the characteristics of the two modernizations that have been made to the instrumentation and control are presented, the first oriented to the installation of three nuclear channels two of wide range and one power channel, renovation of the control console and the installation of the data acquisition system (DAC) cabinet.

The second modernization, which corresponds to the new instrumentation and control of the reactor, is oriented to the change of the control console which supports the control and supervision servers, a nuclear channel NP-1000, printer, four screens of the human interface machine HMI, keyboard of the bar handling system and two keyboards for each of the servers. In addition, the DAC was replaced by the instrumentation cabinet, which includes the reactor protection systems, the redundant control system and the supervision system. The instrumentation and control is characterized by the use of the Ethernet standard to achieve inter-connectivity of the systems, programming of the human machine interface (HMI) using open source code Java ${ }^{\text {tm }}$, and multiplatform, logical separation of functions applying concepts of distributed control and modularity, redundancy, unique failure criteria and independence.

The use of the reactor is shown, referring to the irradiation facilities available for irradiation of materials to be studied using the neutron activation analysis (NAA) technique. Likewise, irradiation is planned to support the use of the fission fingerprint dating technique, research and support to educational institutions through technical conferences and a visit to the nuclear facility.

\section{INTRODUCCIÓN}

$\mathbf{L}$ as necesidades energéticas mundiales han conducido a la investigación de fuentes de energía diferentes de los combustibles fósiles y de otras formas de producción. Luego de que Chadwick descubriera el neutrón, en 1932, y de que Hahn y Strassman descubrieran en 1939 la fisión nuclear, se abrió el camino a una nueva fuente de energía. Los materiales básicos que pueden emplearse para obtener energía nuclear por fisión son principalmente los elementos uranio y torio [1], [2].

A partir de 1939, durante la Segunda Guerra Mundial, los Estados Unidos de Norteamérica adelantaron un proyecto denominado Manhattan, cuya finalidad era extraer la energía presente en el núcleo de los átomos. El físico italiano Enrico
Fermi se vinculó a este proyecto y dirigió la construcción de una pila de uranio en una de las canchas de squash situadas en el piso bajo del estadio Stagg Field de la Universidad de Chicago. La pila llegó a criticidad el 2 de diciembre de 1942, dando lugar al nacimiento del primer reactor nuclear [2], [3].

Luego de 1945, año en que finalizó la Segunda Guerra Mundial, sobrevino el periodo de la Guerra Fría, en cuyo contexto, el 8 de diciembre de 1953, el presidente de Estados Unidos de Norteamérica, general Dwight D. Eisenhower, pronunció ante la Asamblea General de las Naciones Unidas, en Nueva York, el histórico discurso titulado "Átomos para la paz". Este discurso reflejaba un cambio de enfoque, pues se pasaba de la rivalidad entre Estados Unidos y la Unión Soviética al concepto de humanidad-armamento. Es así como, en lugar de centrarse exclusivamente en los peligros de la guerra 
atómica, Eisenhower alabó las aplicaciones nucleares civiles en la agricultura, la medicina y la generación de energía, y propuso establecer un "organismo internacional de energía atómica" que promoviese los usos pacíficos de la energía nuclear "en beneficio de toda la humanidad". Este organismo es el que hoy conocemos como Organismo Internacional de Energía Atómica (OIEA) [3], [4].

La primera acción concreta del presidente Eisenhower fue ofrecer tratados bilaterales de cooperación técnico-científica nuclear a distintos países. Colombia firmó el correspondiente tratado el 5 de mayo de 1955. Fue así como el 11 de junio de ese mismo año Estados Unidos ofreció a Colombia un reactor nuclear tipo piscina de $10 \mathrm{~kW}$ de potencia, que funcionaba con dos kilogramos de uranio ${ }^{235} \mathrm{U}$ al $90 \%$ de enriquecimiento. Este es el reactor nuclear IAN-R1, que alcanzó criticidad el 20 de enero de 1965 y fue inaugurado un mes después por el entonces presidente de Colombia, Guillermo León Valencia [5], [6].

En las siguientes líneas el lector encontrará la descripción de la configuración inicial del reactor IAN-R1 y la manera como se fueron desarrollado y fortaleciendo sus capacidades tecnológicas para darle continuidad a su utilización.

\section{CARACTERÍSTICAS INICIALES DEL REACTOR}

El reactor nuclear IAN-R1 fue diseñado y construido por la compañía Lockheed Western Export Company, y fue operado por el Instituto de Asuntos Nucleares (IAN).

Se trataba de un reactor de investigación heterogéneo de $10 \mathrm{~kW}$ de potencia térmica nominal, tipo piscina, que estaba constituida por un tanque de 1,98 metros de diámetro y 5,28 metros de profundidad. La cubierta del tanque estaba construida en acero al carbón con un espesor de 0,0063 metros, y la superficie interna estaba completamente recubierta por una capa poliestérica de color blanco.

El blindaje biológico estaba conformado por una masa monolítica de concreto convencional de 1,98 metros de espesor situada a nivel de su base octogonal, que se levantaba en forma escalonada terminando en un tercer nivel en forma cilíndrica.

El tanque del reactor funcionaba lleno de agua liviana desmineralizada, que cumplía funciones de moderación de neutrones, blindaje contra las radiaciones y refrigeración del núcleo. La calidad del agua se controlaba por medio de un sistema de tratamiento conexo a la piscina del reactor.

El índice máximo de exposición alrededor del reactor no era superior a $20 \mu \mathrm{Sv} /$ hora, lo cual hacia que el personal científico y en entrenamiento tuviera acceso a todas las áreas sin riesgo de sobreexposición a las radiaciones ionizantes [7], [8].

La potencia inicial de operación fue incrementada, y desde comienzos de 1967 el reactor operó a 20 kW, con periodos cortos de operación a $30 \mathrm{~kW}$, condición que se mantuvo hasta 1975. Este incremento de potencia está ligado al incremento del flujo neutrónico necesario para la producción de radioisótopos.

\subsection{Núcleo del reactor}

El combustible del núcleo estaba constituido por elementos tipo MTR (material testing reactor) en forma de placas planas, que estaban compuestos de uranio metálico enriquecido al $90 \%$ en el isótopo uranio- 235 .

El combustible tenía una aleación y recubrimiento con aluminio tipo 1100, que conformaba una masa crítica de 2,22 kg, distribuidos en dieciséis elementos combustibles, de los cuales trece eran estándar y tres de control, en un arreglo de $4 \times 4$.

Cada elemento estándar estaba conformado por diez placas atornilladas y colocadas dentro de una caja de aluminio. Los elementos de control contenían solo seis placas, con el fin de permitir la introducción de las barras de control.

Los elementos reflectores estaban conformados por veinte elementos de grafito recubiertos por una resina poliestérica impermeabilizante. Para el arranque (inicio de la fisión nuclear) se utilizaba una fuente de neutrones de plutonio-berilio $(80 \mathrm{~g} \mathrm{Pu})$ con una actividad de $5 \mathrm{Ci}\left(1,85 \times 10^{11} \mathrm{~Bq}\right)$ y una intensidad de $10^{6} \mathrm{n} / \mathrm{s}$, que se ubicaba adjunto al núcleo.

El reactor contaba con dos barras de seguridad de acero inoxidable y boro unidas mediante embragues electromagnéticos a mecanismos de accionamiento con movimiento vertical que las introducían o extraían del núcleo a una rapidez de $1,86 \times 10^{-3} \mathrm{~m} / \mathrm{s}$. En caso de emergencia era posible cortar la corriente que alimentaba las bobinas de los embragues, para que las barras del reactor cayeran por acción de la gravedad, lo que llevaba al reactor a un estado seguro y apagado. 
Una tercera barra en acero inoxidable y boro estaba unida a un servomecanismo de movimiento vertical que la introducía o extraía del núcleo a una velocidad de $4,49 \times 10^{-1}$ $\mathrm{m} / \mathrm{s}$, y que permitía el control y los ajustes finos de potencia.

El núcleo, los elementos reflectores, las tres barras de control y tres detectores de neutrones se encontraban inmersos en el fondo del tanque del reactor. El núcleo descansaba sobre una grilla de soporte que estaba sujeta al fondo del tanque del reactor en su parte central. El movimiento de combustible nuclear se hacía con herramientas de manejo suministradas por el fabricante, con la ayuda de un bastidor de almacenamiento.

El reactor fue diseñado para operar con un exceso de reactividad, valor de barras de seguridad, coeficiente de temperatura y demás parámetros, tal como se muestra en la tabla 1 [7], [8].

Tabla 1. Parámetros físicos del núcleo del reactor [7]

\begin{tabular}{ll}
\hline \multicolumn{1}{c}{ Exceso de reactividad } & \multicolumn{1}{c}{$0,6 \Delta \mathrm{k} / \mathrm{k}$} \\
\hline Carga del núcleo & $2,22 \mathrm{~kg}{ }^{235} \mathrm{U}$ (enriquecimiento 90\%) \\
Valor total de las barras de seguridad & $-5,6 \% \Delta \mathrm{k} / \mathrm{k}$ \\
Valor total de la barra reguladora & $-0,4 \% \Delta \mathrm{k} / \mathrm{k}$ \\
Promedio de flujo de neutrones & $1,2 \times 10^{11} \mathrm{~cm}^{-2} \cdot \mathrm{s}^{-1}$ \\
térmicos & $3 \times 10^{11} \mathrm{~cm}^{-2} \cdot \mathrm{s}^{-1}$ \\
Pico de flujo de neutrones térmicos & $1,9 \times 10^{-4} \Delta \mathrm{k} / \mathrm{k} \mathrm{por}{ }^{\circ} \mathrm{C}$ \\
Coeficiente de temperatura $\left(20^{\circ} \mathrm{C}\right)$ & $5,4 \times 10^{-5} \mathrm{~s}$ \\
\hline Vida media promedio de los neutrones &
\end{tabular}

Fuente: autores

\subsection{Facilidades experimentales}

Se emplearon seis posiciones en la placa de rejilla, a un lado del núcleo, para situar los elementos de producción de radioisótopos especiales. Estos elementos eran idénticos a los elementos reflectores de grafito, excepto en que tenían un hueco de acceso central para acomodar muestras de $1 \frac{1}{2} \mathrm{pul}$ gadas $\left(3,81 \times 10^{-2} \mathrm{~m}\right)$ de diámetro y hasta 24 pulgadas $(6,09$ $\times 10^{-1} \mathrm{~m}$ ) de longitud.

Dos tubos de haces neutrónicos confinaban dos caras del núcleo de manera radial y estaban enfocados hacia el centro del núcleo para proveer máximo flujo neutrónico, para fines de irradiación. Fueron suministrados dos tapones de plomo y concreto para blindaje y dos bloques de grafito para fines experimentales.
Además de la adquisición del reactor, el Instituto de Asuntos Nucleares compró e instaló un sistema neumático de transferencia de muestras para ser irradiadas con neutrones térmicos en la parte posterior del núcleo [8].

\subsection{Instrumentación y control}

La instrumentación y el control (I\&C) original del reactor estaban conformados por cuatro canales nucleares, sistemas de paro automático, controles de operación, sistema anunciador y de alarma, barras de control de compensación, seguridad y regulación, monitores de área, consola de control y mecanismos de las barras de control [8].

\subsubsection{Canales nucleares}

Existían cuatro canales nucleares, tres de operación y un canal de seguridad.

El canal de arranque usaba como detector una cámara de fisión, y su intervalo de medida estaba comprendido entre 1 y $10^{4}$ cuentas/segundo. Un interruptor de bloqueo en el intensímetro logarítmico impedía el arranque cuando la intensidad de la fuente de neutrones estaba por debajo de dos cuentas por segundo.

El canal de periodo Log- $\mathrm{N}$ indicaba el nivel de potencia del reactor en un intervalo que iba de $10^{-5}$ hasta tres veces el nivel proyectado de potencia. Debido a que el valor de potencia era de $10 \mathrm{~kW}$, la indicación iba de 100 milivatios a 30 kilovatios; este canal apagaba de emergencia el reactor en caso de periodo corto.

El canal de nivel lineal era capaz de medir el flujo neutrónico en un intervalo de operación del reactor desde 100 milivatios hasta 1 megavatio, y usaba como detector una cámara de ionización compensada (CIC) acoplada a un micro-amperímetro. Este canal permitía leer desde el momento del arranque hasta plena potencia mediante el ajuste de un interruptor que operaba entre $10^{-11}$ y $10^{-3}$ amperios.

El canal de seguridad utilizaba una cámara de ionización no compensada que trabajaba en un intervalo comprendido entre 0 y 1,5 veces la potencia proyectada.

\subsubsection{Sistemas de paro del reactor}

Era un sistema que controlaba tres tipos de acción automáticas o manuales para corregir condiciones anormales o indebidas, a saber: apagado de emergencia rápido o por corto periodo, apagado de emergencia lento (producido por alto 
voltaje, sensores de los sistemas periféricos, pulsador de apagado manual, interruptor de llave, alto nivel en los canales Log-N y de nivel lineal) y caída del grupo de barras por acción del operador desde la consola.

\subsubsection{Controles de operación}

La fuente de neutrones estaba colocada en un portafuentes contiguo al núcleo. Para asegurar que la fuente estuviera en la debida posición para el arranque del reactor, un interruptor situado en el intensímetro logarítmico evitaba que las barras de control se subieran, a no ser que el ritmo de contaje fuera superior a dos cuentas/segundo.

Había un sistema de control automático para operar el reactor, que movía la barra reguladora hasta que alcanzara un punto determinado sobre el inscriptor lineal de potencia.

\subsubsection{Sistema anunciador y de alarma}

Cuando se presentaba un causal de apagado de emergencia, o cuando surgía otro problema, sonaba una alarma, y en la consola de control se encendía una luz que indicaba el evento iniciante del problema.

\subsubsection{Barras de control}

Se usaban dos barras de seguridad y una barra de control fino de potencia que eran accionadas manualmente con interruptores. En la consola se visualizaba el acople magnético de cada barra, intervalo de compensación, límite superior y límite inferior de las barras.

Las posiciones de las barras se indicaban tanto en una escala vertical como en un dispositivo digital de lectura fina que tenía una resolución de $1 \times 10^{-4} \mathrm{~m}$. La barra reguladora se movía manualmente en un intervalo comprendido entre 100 milivatios y 10 kilovatios.

\subsubsection{Monitores de área}

Fueron suministrados seis monitores de área tipo centelleo para vigilar el nivel de radiación. Había un monitor sobre la piscina del reactor, dos para el desmineralizador del sistema de tratamiento de agua y tres en la consola. Estos monitores podían ser graduados para producir el apagado del reactor por exceder los valores predeterminados.

\subsubsection{Consola de control}

La consola de control había sido diseñada para dar máxima visibilidad sobre ella y tenía facilidad de acceso a los controles e indicadores, que estaban situados en un solo grupo frente al operador. Los colores de las luces indicadoras en la consola mostraban al operador la situación del reactor en un solo vistazo. Tanto los procedimientos de operación como las interconexiones evitaban que el operador sacara las barras de control cuando había señal de una luz roja (figura 1).

\subsubsection{Mecanismos de accionamiento de las barras de control}

Estos mecanismos fueron fabricados por la empresa Diamond Power Specialty Corporation, tenían forma tubular y estaban situados en una posición vertical sobre el núcleo. Cada barra de control era movida mediante una fuerza hacia arriba sobre un tubo de extensión que estaba acoplado a un electromagneto (excepto en el caso de la barra reguladora) y a un mecanismo de tornillo movido por un motor situado en el extremo superior del tubo. Todos los mecanismos que accionaban arriba y abajo las tres barras de control estaban provistos de motores de inducción de reversión instantánea.

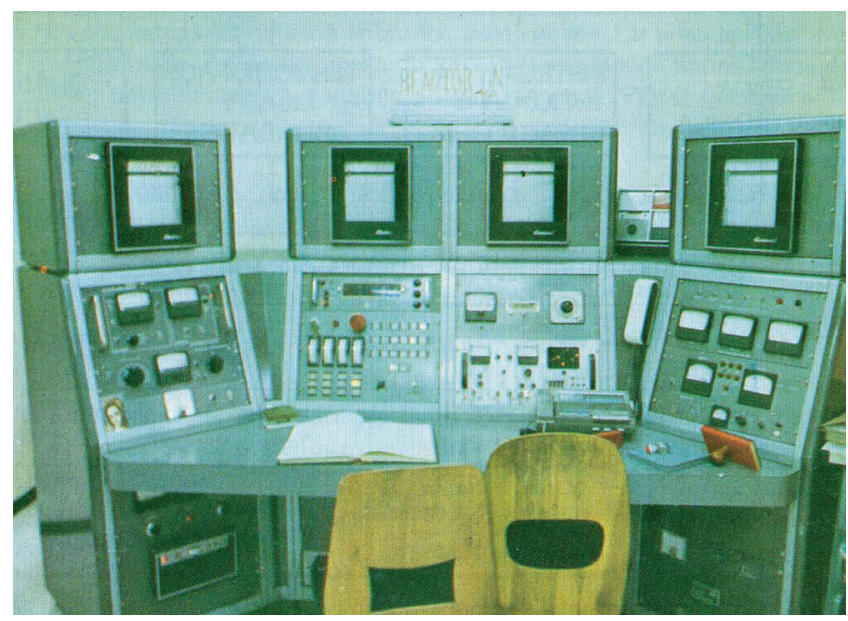

Figura 1. Consola del reactor. Fuente: [8] 


\subsection{Sistema de tratamiento y refrigeración de agua}

Este sistema de tratamiento de agua, que tiene funciones de purificación y refrigeración, limitaba la velocidad de corrosión del sistema, la acumulación de productos de corrosión y la activación del refrigerante mediante el empleo de microfiltros y de resinas de intercambio iónico. Bajo apropiadas condiciones de operación, la pureza del agua se podía mantener en 2 ppm en términos de $\mathrm{CaCo} 3$, y el PH en 6,5. La capacidad de extracción de calor dada por el intercambiador de calor era de 10 550,56 watts/hora, y se había calculado para mantener la temperatura de la piscina o tanque del reactor en 30 grados Celsius durante operación continua de 10 kilovatios.

Adicionalmente, un sistema de tratamiento de agua se encuentra instalado en una piscina o tanque de decaimiento radiactivo que fue instalado como sistema auxiliar, localizado en una edificación adjunta y que se comunica con el recinto del reactor. Este tanque está lleno de agua de las mismas condiciones que la utilizada en el tanque del reactor hacia donde es posible hacer bombeo, en caso de que el tanque del reactor sufra pérdida de agua. Otra de las funciones de la piscina de decaimiento es depositar combustible nuclear irradiado u objetos activados.

\section{PRIMERA MODERNIZACIÓN DE LA INSTRUMENTACIÓN Y CONTROL DEL REACTOR}

Teniendo en cuenta el avance tecnológico, en el año 1987 el IAN observó que la instrumentación y el control originales del reactor habían quedado obsoletos, ya que el equipo había sido diseñado con tecnología electrónica de tubos de vacío y necesitaba mantenimiento y calibración, lo cual, unido a la carencia e imposibilidad de conseguir partes de repuesto, estaba ocasionando un rápido deterioro de su confiabilidad. Entonces se inició un programa de modernización ajustado a los siguientes criterios y requerimientos [9]:

» La instrumentación original estaba culminando su tiempo de vida útil, ya que tenía más de veinticinco años de operación, y era imposible adquirir equipos de reemplazo, ya que su fabricación había sido descontinuada.

ı La consola de control había sido diseñada con tecnología electrónica de tubos de vacío, que no permitía la renovación parcial de equipos o circuitos por la

incompatibilidad con la tecnología del momento, basada en semiconductores.

》)

)

$\gg$ ción y el control originales no satisfacía la nueva filosofía aplicada a reactores de investigación, la cual había sido ajustada.

〉 dad en las mediciones, ocasionadas por el envejecimiento de los equipos, producían una disminución de la confiabilidad de la instrumentación.

\Se requería ejecutar proyectos de incremento de potencia de operación del reactor, lo cual se dificultaba por las limitaciones funcionales y el rango de operación de la instrumentación.

\subsection{Modernización realizada}

La consola de comando de operaciones y la nueva instrumentación y control del reactor, que pasamos a describir, fue fabricada por la General Atomics [9].

\subsubsection{Canales nucleares}

Se pusieron en marcha tres canales nucleares: dos de amplio rango modelo NM-1000 y un canal de potencia modelo NPP-1000.

El canal de potencia NPP-1000 usaba como detector una cámara de ionización y era utilizado para monitorear gráficamente la forma del pulso de potencia del reactor, de acuerdo con el modo de operación seleccionado.

Los canales de amplio rango modelo NM-1000 usaban como detectores cámaras de fisión $\left({ }^{235} \mathrm{U}\right)$, y su rango de detección iba desde el nivel fuente hasta el $120 \%$ de la potencia nominal.

\subsubsection{Consola de control}

La nueva consola permitía al operador controlar y monitorear el estado de los parámetros y variables de operación del reactor, disponía de un sistema de selección de modo de operación manual y automático, de un conjunto de interruptores para el manejo de los mecanismos de accionamiento de las barras de control, teclado de computador, una impresora, monitores para visualización de potencia de operación - que se presentaba en forma lineal, porcentual y logarítmi- 
ca一, señal de período, posición de mecanismos de las barras de control, temperatura del agua y de algunos elementos combustibles del reactor. Además, los monitores permitían ver el valor de las variables y los parámetros de operación, el estado de las señales indicadoras de límites de prevención y el estado de los dispositivos de seguridad (figura 2). Adicionalmente, el computador de la consola permitía grabar toda la información de la operación en un archivo histórico.

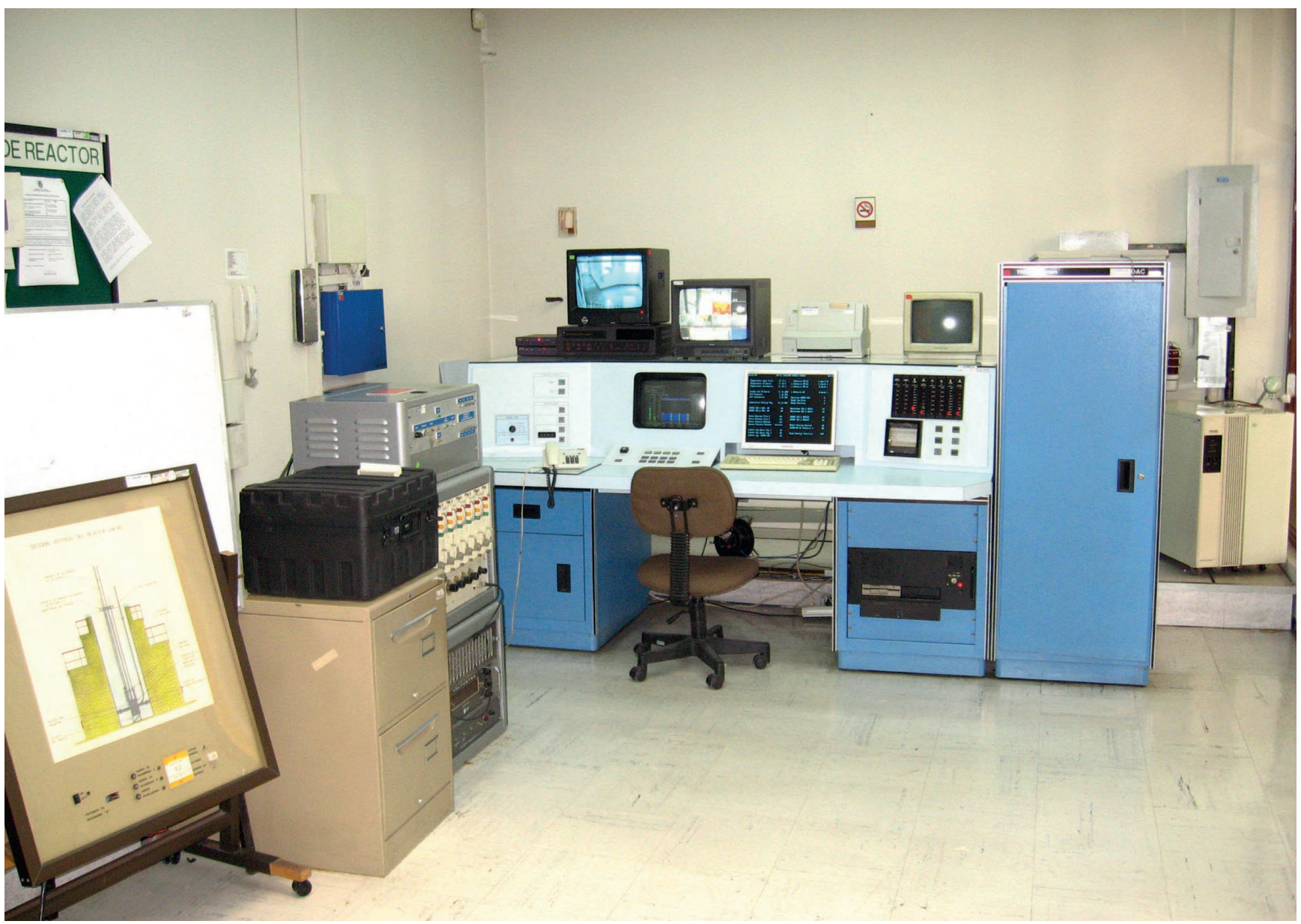

Figura 2. Instrumentación y control usados a partir de 1996.

Fuente: [9]

\subsubsection{Sistema de adquisición de datos (DAC)}

Estaba constituido por un conjunto de sistemas electrónicos controlados por computador. Se encargaba de registrar, almacenar y procesar la información de todas las variables y los parámetros de operación del reactor y de establecer la interface de comunicación interactiva con la consola de control del reactor.

Los componentes del DAC estaban distribuidos por niveles, así:

Nivel 1: sistema de distribución de potencia.

Nivel 2: sistema de procesamiento y conversión para señales de entrada y salida AC.
Nivel 3: sistema de procesamiento y conversión para señales de entrada y salida DC.

Nivel 4: módulos de acondicionamiento de diversas señales provenientes de algunos sistemas de medición, tales como temperatura del combustible, temperatura de refrigerante, flujo de agua en el sistema de refrigeración, conductividad del agua del reactor, voltaje de los magnetos de los mecanismos de las barras de control y, además, señales de ajuste de límites de nivel de prueba y calibración de circuitos.

Nivel 5: sistema de control manual y automático de la barra reguladora y del DAC.

Nivel 6: computador del DAC. 
El DAC procesaba la información proporcionada por los canales nucleares NM-1000, por los mecanismos de accionamiento de las barras de control, por el sistema de protección radiológica, los medidores de temperatura, los medidores del caudal de refrigerante, el medidor de conductividad, la fuente de voltaje de los magnetos y las señales de comando provenientes de la consola para el control de la operación del reactor. La mayoría de los componentes del circuito de apagado de emergencia del reactor estaban localizados en el DAC.

Luego de esta primera modernización de la instrumentación, el reactor fue operado por el Instituto de Ciencias Nucleares y Energías Alternativas (INEA) nombre que adoptó el IAN después de ser reestructurado [10], y que fue liquidado en 1997 [11]. En ese mismo año el INEA realizo el cambio de combustible nuclear del reactor, pasando de tecnología MTR de alto enriquecimiento (90\%) a TRIGA (acrónimo de training, research, isotopes) de bajo enriquecimiento (20\%), reactor nuclear diseñado y fabricado por la General Atomics de Estados Unidos. Además, realizó el cambio del sistema de refrigeración con capacidad para $100 \mathrm{~kW}$ de operación y el sistema de procesamiento de agua del reactor [12].

El reactor con núcleo nuevo alcanzó criticidad el 29 de agosto de 1997 y operó a potencia de $30 \mathrm{~kW}$ en forma regular desde el 2 de octubre de 1997 hasta el 31 de marzo de 1998, fecha en que entró en parada prolongada debido el cierre definitivo del INEA.

La configuración del nuevo núcleo TRIGA y su posición de irradiación de muestras por gradilla se presenta en la figura 3, y las características operacionales se presentan en la tabla 2 [13].

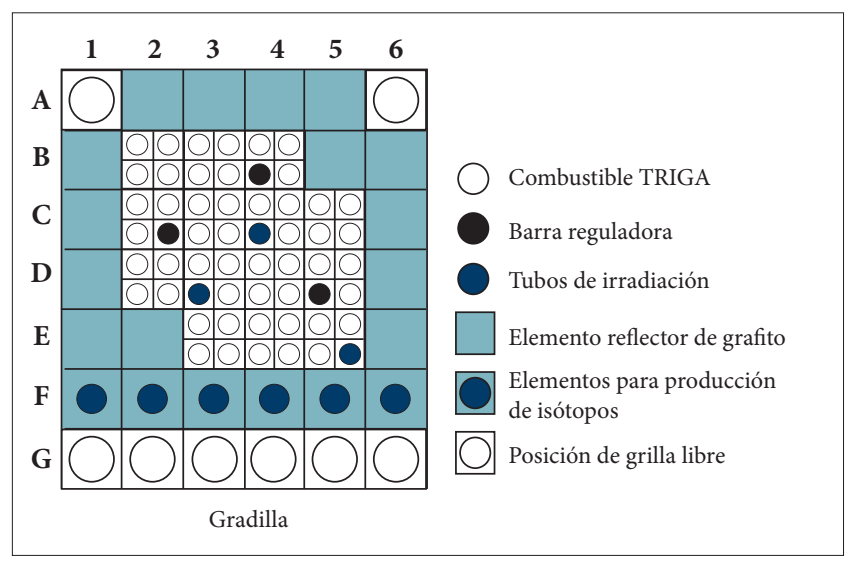

Figura 3. Nuevo núcleo TRIGA del reactor. Fuente: autores

\section{SEGUNDA MODERNIZACIÓN DE LA INSTRUMENTACIÓN Y EL CONTROL DEL REACTOR}

Por disposición del Decreto n. ${ }^{\circ}$ de 1452 de 1998, la operación del reactor fue transferida al Instituto de Investigaciones en Geociencias, Minería y Química (Ingeominas), hoy Servicio Geológico Colombiano (SGC) [14].

Con el propósito de hacer mantenimiento correctivo del tanque de la piscina del reactor, el 6 de marzo de 2006 se inició el traslado de la totalidad del núcleo del reactor, catorce elementos combustibles y veinte reflectores de grafito hacia la piscina de decaimiento. Finalizado el mantenimiento, el núcleo del reactor fue retornado al tanque del reactor, y el 21 de octubre de 2005 el reactor fue puesto a crítico una vez más [15].

Nuevamente, y después de veinte años, esta vez el SGC observó que la instrumentación y el control eran obsoletos y exigían mantenimiento y calibración, lo cual, unido a la carencia de partes de repuesto y la imposibilidad de conseguirlas, estaba ocasionando un rápido deterioro de su confiabilidad. Fue así como se tomó la decisión de modernizar la I\&C del reactor, en el marco del Convenio Especial de Cooperación n. ${ }^{\circ}$ 716-2009, suscrito entre el Departamento Administrativo de Ciencia, Tecnología e Innovación (Colciencias), el Centro Internacional de Física (CIF) y el Servicio Geológico Colombiano, convenio que contaba con el soporte del Instituto Colombiano de Petróleo (ICP) [16].

Esta segunda modernización fue realizada de acuerdo a los siguientes criterios y requerimientos:

ı La instrumentación fabricada por la General Atomics estaba culminando su tiempo de vida útil, ya que tenía más de veinte años de operación, y era imposible adquirir equipos de reemplazo, ya que su fabricación había sido descontinuada.
〉

$\gg$

〉)
Había un inventario limitado de partes de repuesto.

La tasa de falla y descalibración de equipos era muy alta.

Actualización de la nueva instrumentación a la filosofía de seguridad y control de reactores de investigación.

Alto grado de especialización de la instrumentación, que se traducía en un alto costo de operación y, en caso de falla, en una baja disponibilidad de la instalación. 
Tabla 2. Características operacionales del núcleo TRIGA [13]

\begin{tabular}{|c|c|}
\hline Carga crítica aproximada & 48,51 barras $\left(2,49 \mathrm{~kg}^{235 \mathrm{U}}\right)$ \\
\hline Carga aproximada de operación & 50 barras \\
\hline$ß e f f(\Delta \mathrm{k})$ & 0,0073 \\
\hline$\ell$, vida del neutrón & $45 \mathrm{~ms}$ \\
\hline $\begin{array}{l}\alpha \text {, coeficiente de realimentación } \\
\text { negativo pronto de reactividad por } \\
\text { temperatura, promedio } 23^{\circ} \mathrm{C}->400^{\circ} \mathrm{C}\end{array}$ & $9,3 \times 10^{-5}$ \\
\hline $\begin{array}{l}\text { Exceso de reactividad máximo de } \\
\text { diseño }\end{array}$ & $1,83 \%(\$ 2,50)$ \\
\hline $\begin{array}{l}\text { Valor mínimo del sistema de control } \\
\text { con barra de máximo valor de } \\
\text { reactividad fuera }\end{array}$ & $2,50 \%(\$ 3,42)$ \\
\hline Potencia & $30 \mathrm{~kW}$ \\
\hline $\begin{array}{l}\text { Temperatura máxima aproximada del } \\
\text { combustible }\end{array}$ & $145^{\circ} \mathrm{C}$ \\
\hline $\begin{array}{l}\text { Valores operacionales de reactividad } \\
\text { y cambio de reactividad frío-caliente } \\
\text { (aprox.) }\end{array}$ & $0,22 \%(\$ 0,30)$ \\
\hline Valor del Xe en equilibrio & $0,11 \%(\$ 0,15)$ \\
\hline Experimentos (aprox.) & $0,73 \%(\$ 1,00)$ \\
\hline Total & $1,06 \%(\$ 1,45)$ \\
\hline
\end{tabular}

Fuente: autores

\subsection{Segunda modernización}

La nueva instrumentación y control del reactor fueron implementados por el Instituto Nacional de Investigaciones Nucleares (ININ) de México, país en el cual también fue fabricada la nueva consola de operaciones, que fue instalada y puesta en marcha en 2012. Esta instrumentación es la que se utiliza actualmente, y está enfocada a reducir la dependencia respecto del fabricante original y abrirla con una estructura modular para facilitar futuras actualizaciones. Dicha instrumentación tiene las siguientes características [17]:

» Separación lógica de funciones aplicando conceptos de control distribuido y modularidad.

》 Redundancia, criterio de falla única e independencia.

» Cableado directo del sistema de protección, que evita realizar funciones de seguridad mediante el software.

» Empleo del estándar Ethernet para lograr la interconectividad de los sistemas, lo cual reduce el cableado de señales desde y hacia el reactor y la consola.

»Cuatro pantallas comerciales de alta resolución en la interface humano-máquina (HMI) para muestra panorámica de los parámetros de operación y control del reactor.

» Servidores comerciales de uso industrial (sin tarjetas de función especializada), de fácil sustitución o actualización.

» Transferencia tecnológica mediante planos, códigos fuente, manuales y capacitación, con posibilidad de hacer actualizaciones por el usuario.

» Programación de la HMI empleando código de fuente abierta Java $^{\text {tm }}$, y multiplataforma. Esto permitirá disminuir costos y dependencias en actualizaciones futuras, que podrán ser realizadas por el usuario.

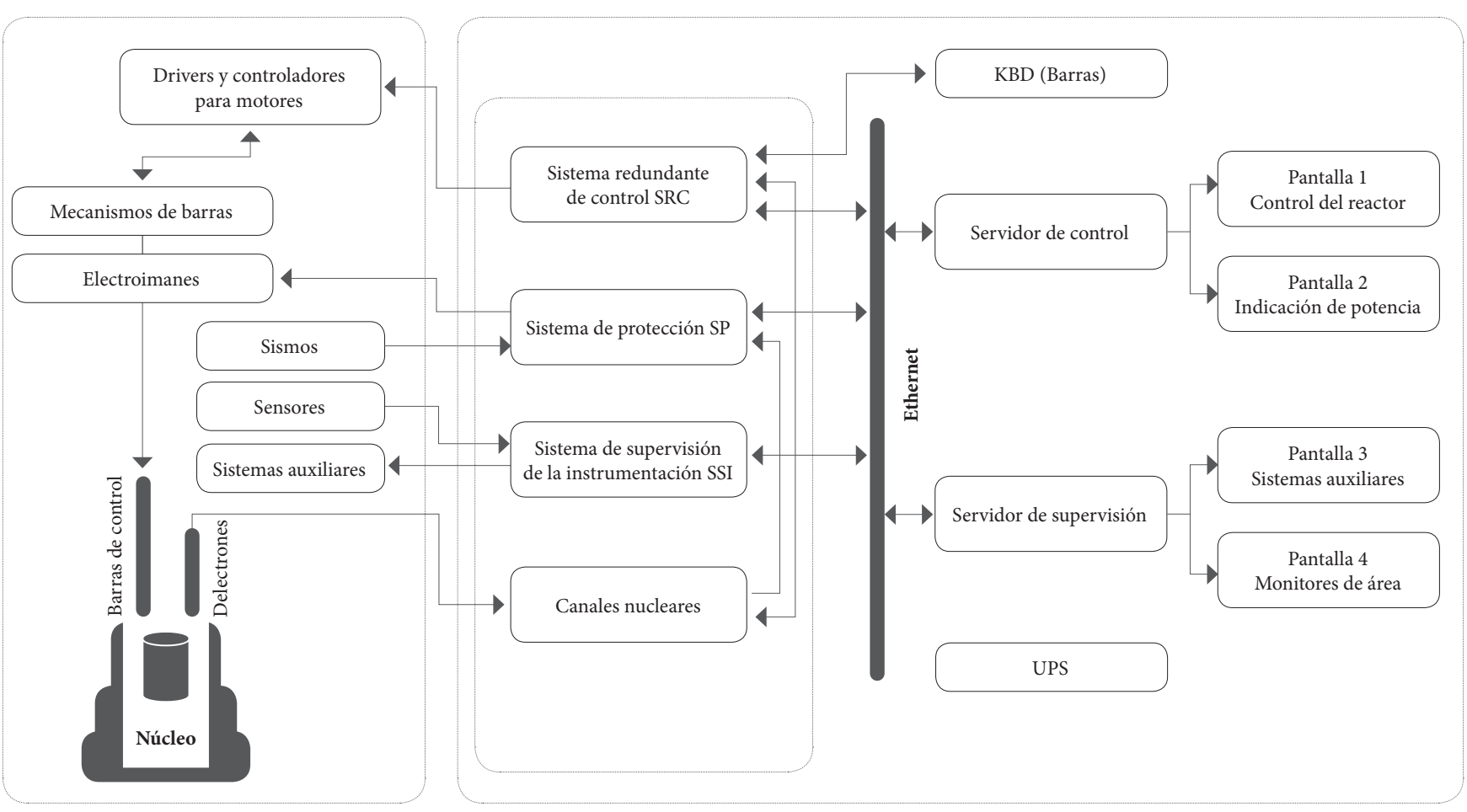

Figura 4. Instrumentación y control actuales.

Fuente: [18] 
Los componentes de esta nueva I\&C están distribuidos en cuatro niveles, mostrados en la figura 4:

Nivel 1: corresponde al nivel de sensores y actuadores, que interactúan directamente con el reactor.

Nivel 2: son los acondicionadores y manejadores de señal, canales nucleares y de protección.

Nivel 3: sistema de adquisición de datos y control.

Nivel 4: interacción humano-máquina (HMI).

\subsubsection{Sistema redundante de control (SRC)}

Se encarga de los modos de operación manual y automático del reactor, para lo cual se mueve la posición de las barras de control a través del sistema de manejo de barras (SMB). Se encarga también de la adquisición de la posición de las barras, los interbloqueos para operación del reactor, y controla la potencia y el periodo a través de los canales nucleares.
El SRC consta de un sistema de control lógico programable (PLC) de arquitectura modular-redundante, y tiene un PLC de respaldo, o secundario, cuya configuración es idéntica a la del primario. Mediante la detección y la respuesta programada, el sistema redundante puede realizar la transición automática del controlador primario y sus módulos asociados al controlador secundario y sus módulos idénticos (figura 5) [18].

\subsubsection{Sistema de protección (SP)}

Contiene la lógica de votación y las fuentes de energía de los magnetos de las tres barras; estas últimas cumplen funciones de control y seguridad. Tiene la función de llevar el reactor a un apagado seguro en caso de presentarse alguna de las condiciones de falla preestablecidas.

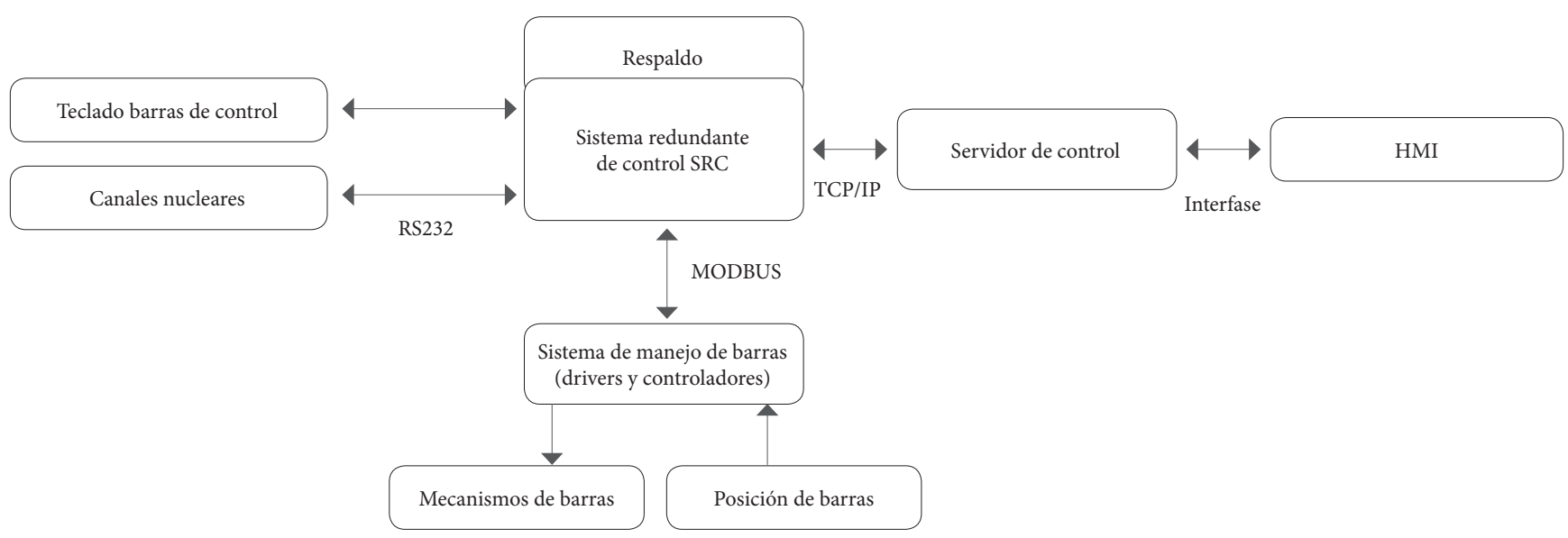

Figura 5. Sistema redundante de control (SRC).

Fuente: [18]

Está formado por dos módulos redundantes y aislados diseñados con un lógica combinacional basada en lógica TTL, y un módulo de control de magnetos de las barras de control.

El SP proporciona un aislamiento funcional de los sistemas restantes, basado en una serie de optoacopladores a la entrada de todas las señales y optoacopladores y relevadores a la salida (figura 7) [19].

\subsubsection{Sistema de supervisión de la instrumentación (SSI)}

Tiene la función de monitorear constantemente las variables de proceso, tanto en operación como en reposo, por medio de la adquisición de datos de los sistemas auxiliares, como son el intercambiador de calor, la temperatura de la piscina y el sistema de monitoreo de área.

El SSI está conformado por un PLC equipado con tarjetas de adquisición de datos y de comunicación, ya que su información es entregada por medio de un bus Ethernet (figura 6) [18]. 


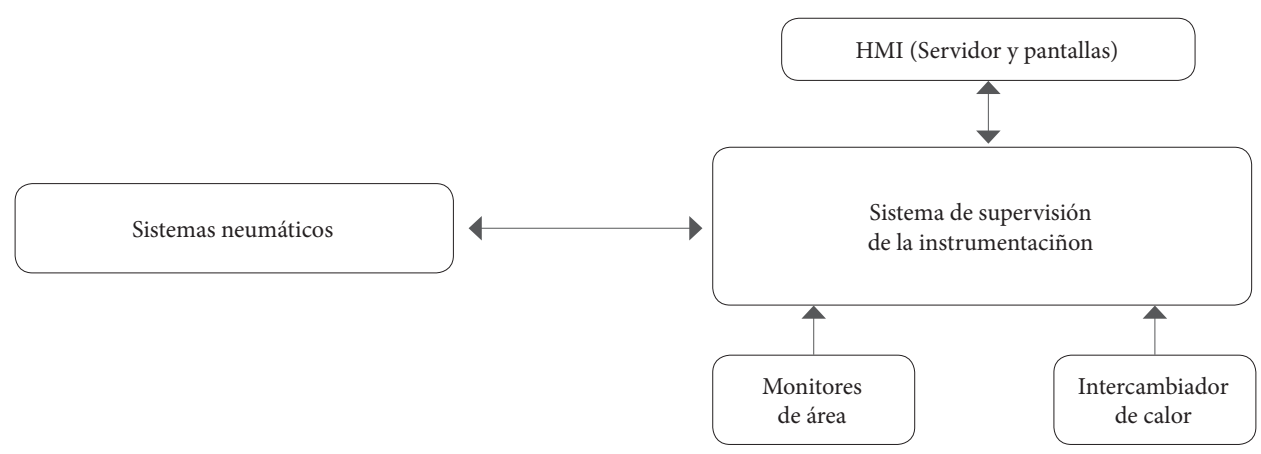

Figura 6. Sistema de supervisión de la instrumentación (SSI).

Fuente: [18]

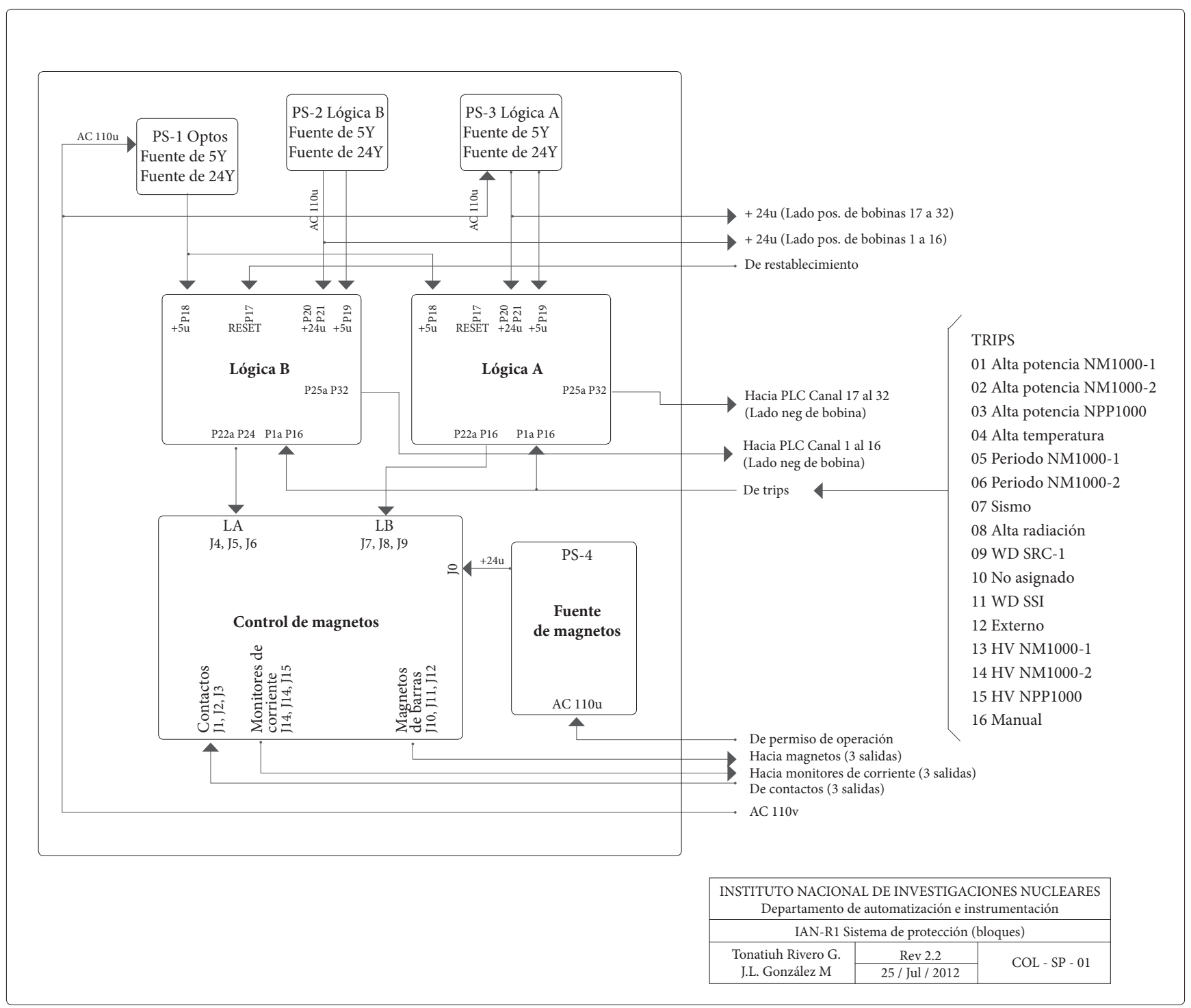

Figura 7. Sistema de protección (SP).

Fuente: [19] 


\subsubsection{Gabinete de instrumentos}

El gabinete es un armario metálico grado de protección IP55 (EN60529), con puerta transparente de cristal templado y un sistema de ventilación que garantiza la disipación de calor dentro del armario. Se encuentra ubicado en la parte posterior de la consola de control y contiene los siguientes elementos (figura 8):

\) El sistema redundante de control (SRC).

» El sistema de supervisión de la instrumentación (SSI).

» El sistema de protección (SP).

\subsection{Canales nucleares}

Actualmente continúan funcionando los dos canales nucleares de amplio rango modelo NM-1000, puestos en marcha en la modernización anterior, y un canal de potencia modelo NP-1000.

\subsubsection{Interfaz humano-maquina (HMI)}

Muestra al operador, por medio de cuatro pantallas, los controles, indicadores, alarmas y mensajes para el control seguro del reactor. Se encarga de realizar las verificaciones y pruebas de los sistemas y le presenta al operador la información necesaria para la toma de decisiones (figura 9) [20].

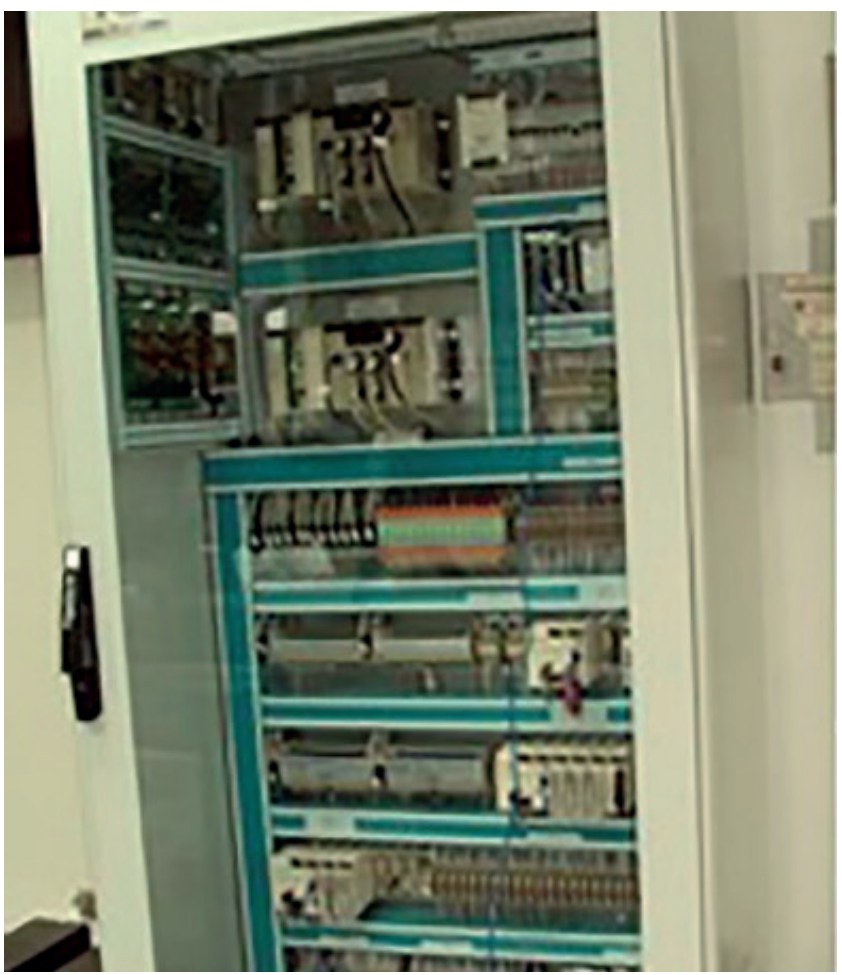

Figura 8. Gabinete de instrumentos. Fuente: [20]

\subsubsection{Consola de operación}

La consola tiene estructura robusta NEMA 12 y está fabricada en lámina de acero al carbón y lámina de acero inoxidable, con cuatro puertas frontales y tres puertas posteriores. Cuenta con charolas deslizables y brazos retráctiles para colocar los monitores de 21,5” de la HMI, acometidas de cables y sistema de ventilación (figura 8). La consola soporta los siguientes elementos:

\Servidores de control y supervisión.

») Canal nuclear NP-1000.

\) Impresora.

\) Cuatro pantallas de la interface HMI.

\D Dos teclados para cada uno de los servidores.

») Teclado del sistema de manejo de barras.

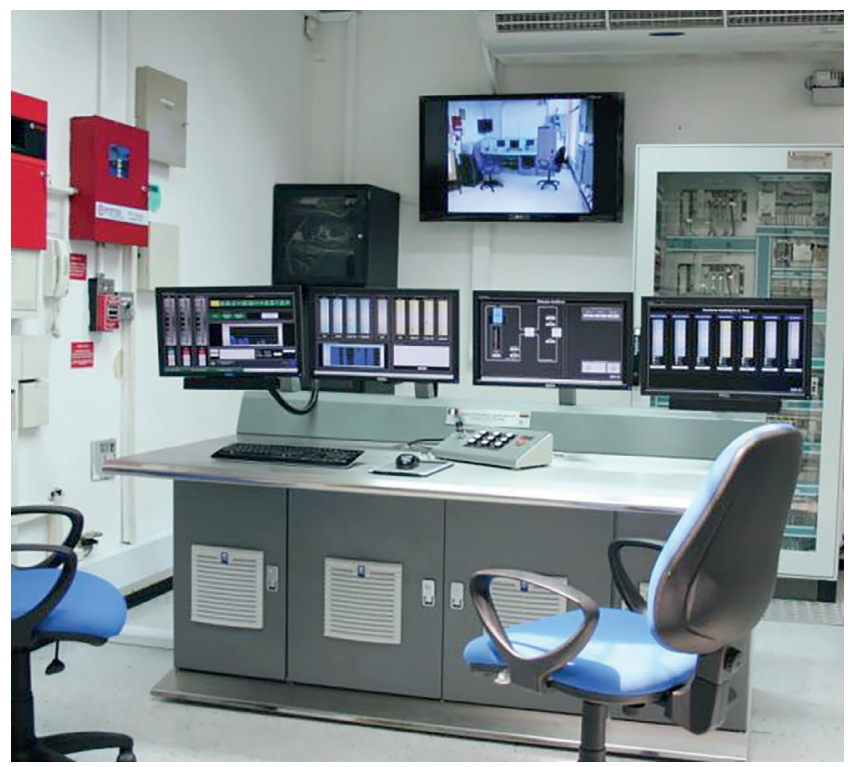

Figura 9. Consola de control usada a partir de 2012. Fuente: [20]

\section{UTILIZACIÓN DEL REACTOR}

Los programas del IAN relacionados con la utilización del reactor eran física nuclear, termohidráulica y blindajes, instrumentación, seguridad nuclear y química. A partir de 1969 se dio lugar a publicaciones en bioquímica, trazadores radiactivos en la industria del petróleo y otras plantas industriales, agricultura, isótopos y energía geotérmica, utilización de algunos radioisótopos y radiografía industrial. De igual manera, se realizaron trabajos en medida de humedad y espesor de suelos, química y radioquímica, utilización de 
radioisótopos en hidrología superficial, y el IAN implementó de forma básica la primera etapa del ciclo de combustible nuclear: la exploración y explotación de uranio.

Hacia 1979 continuó la utilización del reactor para la irradiación de minerales con contenido de uranio y torio, y para la producción de materiales radiactivos, seguido de la producción de oro 198, lantano 140 y bromo 82, realizada en 1983. Ese mismo año el reactor fue ajustado para operar a una potencia de $30 \mathrm{~kW}$, antes de la primera actualización de su instrumentación y control.

Además, se efectuaron estudios para la producción de tecnecio-99m $\left({ }^{99 \mathrm{~m}} \mathrm{Tc}\right)$ mediante la producción de molibdeno irradiado en el reactor, para suministros menores en el campo de la medicina nuclear.

La seguridad radiológica como una disciplina inherente a la utilización del reactor y al manejo de fuentes radiactivas tuvo un desarrollo que comenzó en 1966 con el informe titulado "Reglamento de seguridad radiológica" y posteriores publicaciones, como "La seguridad radiológica para radiografía industrial”, realizado en 1985 [3], [21].

Actualmente, el SGC ha dispuesto seis monitores de área para el monitoreo de campos de radiación en sala de control, boca de tanque, sistema de procesamiento, tubo de haces norte, tubo de haces sur y piscina de decaimiento. Además, se hace seguimiento biológico al personal ocupacionalmente expuesto que se encarga de la operación del reactor. Por consiguiente, es posible irradiar muestras para que sean analizadas mediante la técnica de análisis por activación neutrónica (AAN), haciendo uso de las facilidades de irradiación disponibles, como son: tres posiciones de irradiación dentro del núcleo por sistema neumático nuevo, seis posiciones de irradiación en reflectores de grafito, localización de gradilla frente al núcleo y el sistema neumático original del reactor.

Además, teniendo en cuenta el carácter de institución adscrita al Sistema de Ciencia y Tecnología, el SGC utiliza el reactor para hacer investigación aplicada y apoyar a instituciones educativas mediante conferencias técnicas y visita a la instalación nuclear.

Del mismo modo, el SGC tiene proyectos a corto plazo para la utilización de la técnica de datación por huellas de fisión, metodología útil para obtener indicios sobre la probabilidad de la presencia de hidrocarburos en el lugar de extracción de la muestra.

\section{AUTORIZACIONES}

La autorización para la operación del reactor seguía una línea de mando sujeta a la estructura organizacional del Instituto de Asuntos Nucleares, que estaba conformada por la Junta Directiva, la Dirección General, la Subdirección General y la Secretaría General.

Además, existían catorce áreas técnicas, entre las que se encontraba el Área del Reactor, que estaba conformada por una coordinación de área, de la que dependían los grupos de trabajo en Física Neutrónica, Instrumentación de Reactores, Termohidráulica y Blindajes, Química de Reactores y el Grupo de Seguridad Física, Nuclear y Radiológica, que se encargaba de la operación y supervisión del reactor.

Con el propósito de contribuir a la seguridad nuclear y radiológica, en el IAN se había constituido un comité interno de seguridad del reactor, conformado por los profesionales especializados del área. Este comité tenía como objetivos analizar y evaluar los procedimientos experimentales o modificaciones en los sistemas y equipos que constituían el reactor, y que de alguna manera pudieran afectar los criterios y normas de seguridad establecidos para la operación del reactor nuclear [21].

De acuerdo con recomendaciones realizadas por el OIEA a los países miembros, que fueron realizadas con criterios de independencia e incremento en la seguridad de la operación del reactor, fue creada la Autoridad Reguladora, que funcionaría de manera independiente de la entidad que realizara la operación del reactor. Fue así como, mediante la Resolución n. ${ }^{\circ} 181757$ del 26 de diciembre de 2005 se creó el Grupo de Asuntos Nucleares, del Ministerio de Minas y Energía, que funge como autoridad reguladora nacional [22].

Los tipos de licencia que expide la Autoridad Reguladora para el reactor nuclear son: licencia de operación, licencia de parada prolongada, licencia de modificación y licencia de desmantelamiento. Adicionalmente, todos los funcionarios de grupo de operación del reactor deben certificarse en protección radiológica ante dicha autoridad, de acuerdo al rol que desempeñan, y adicionalmente se deben certificar como supervisores y operadores los aspirantes a desempeñar estos roles [23].

Además de las licencias y certificaciones expedidas por la Autoridad Reguladora, la operación del reactor se realiza de acuerdo con la línea de mando de la estructura 
organizacional del Servicio Geológico Colombiano, que está conformada por el Consejo Directivo, la Dirección General y siete direcciones técnicas, entre ellas la Dirección de Asuntos Nucleares, de la cual depende el grupo encargado de la operación del reactor nuclear [24].

Adicionalmente, el SGC creó el Comité de Seguridad del reactor nuclear de investigación IAN-R1, que está conformado por dos expertos en protección radiológica y seguridad nuclear de nacionalidad colombiana y un experto en reactores nucleares de investigación, de origen extranjero. El propósito de este comité es contribuir a la seguridad nuclear y radiológica del reactor mediante la emisión de conceptos de seguridad relacionados con los cambios de configuración del núcleo, la revisión de los límites y las condiciones operacionales, las recomendaciones sobre los aspectos de seguridad asociados a las modificaciones de la instrumentación y control, recomendaciones sobre alternativas de desmantelamiento y seguridad en experimentos [25].

La autorización para operar el reactor también está ligada al cumplimiento de los compromisos internacionales. Es así como el SGC, como operador del reactor nuclear, ha venido cumpliendo con los convenios y tratados internacionales suscritos por Colombia, incluidas las salvaguardias nucleares realizadas por el Organismo Internacional de Energía Atómica [26].

\subsection{Recursos humanos}

El reactor nuclear IAN R1 fue operado desde el comienzo por personal colombiano, con asesoría de expertos del Organismo Internacional de Energía Atómica. El equipo que intervino fue un supervisor, un supervisor asistente, un operador y su asistente, un físico de seguridad, el director de experimentos y su asistente, dos personas encargadas del manejo de combustible, una persona que se ocupaba de la carga de combustible y una persona dedicada al manejo de la instrumentación [27].

En 1997 el grupo de operación del reactor estaba conformado por el jefe de reactor, un supervisor, un operador y una persona encargada del mantenimiento. Este personal, excepto la persona encargada del mantenimiento, fue desvinculado del reactor debido al cierre del INEA.
Actualmente, la operación del reactor se realiza con un equipo permanente compuesto por un supervisor, un operador, un oficial de protección radiológica y dos personas de mantenimiento. Con el propósito de asegurar la continuidad de la operación, un segundo supervisor está en proceso de certificación, y un segundo operador está en formación.

\section{CONCLUSIONES}

Los aspectos políticos y el enfoque bélico del uso de la energía nuclear han causado un fuerte impacto en las ciencias nucleares, pero afortunadamente un enfoque pacífico de la utilización de la energía nuclear, como fue propuesto en el discurso "Átomos para la paz", ha constituido un hito histórico para la humanidad, ya que instó al control y la promoción internacionales de la energía atómica y motivó la creación del Organismo Internacional de Energía Atómica (OIEA).

La operación de un reactor nuclear de investigación, como el reactor IAN-R1, permite la producción de radioisótopos que encuentran usos en aplicaciones médicas e industriales, datación de muestras que contribuyen a la exploración de hidrocarburos, análisis para caracterización de materiales, docencia e investigación.

Los recursos humanos disponibles para la operación del reactor han sido fortalecidos, ya que la operación del mismo se realiza con un equipo permanente compuesto por un supervisor, un operador, un oficial de protección radiológica y dos personas de mantenimiento. Con el propósito de asegurar la continuidad de la operación, un segundo supervisor está en proceso de certificación y un segundo operador está en formación.

A pesar del traspié sufrido por las ciencias nucleares debido al cierre del INEA, ha podido resurgir y hoy dichas ciencias se encuentran fortalecidas en el país con un reactor cuyo combustible nuclear es relativamente nuevo, y cuya instrumentación y control nuevos ha evolucionado desde los tubos de vacío hasta la tecnología de controladores lógicos programables (PLC), que cumple con los estándares internacionales de seguridad exigidos a estas instalaciones nucleares. 


\section{Referencias}

[1] S. Glasstone y A. Sensonske, Ingeniería de reactores nucleares, Barcelona: Reverté, 1962.

[2] J. Turner, Atoms, Radiation and Radiation Protection, 3. ${ }^{\mathrm{a}}$ ed., Weinheim: Wiley-VCH Verlag GmbH \& Co. KGaA, 2007.

[3] H. Múnera, Tecnología nuclear no médica en colombia: pasado presente y futuro. Bogotá: Tecnicontrol, 2011.

[4] Agencia Internacional de Energía Atómica (OIEA), "Los átomos para la paz de Eisenhower", Boletín de la OIEA 54-4, dic. 2013.

[5] Instituto de Asuntos Nucleares, Primer registro de operación del reactor IAN-R1, 20 en., 1965.

[6] Ministerio de Relaciones Exteriores de Colombia. Traducción oficial "Acuerdo para la venta del reactor nuclear", Contrato G64-6105. Bogotá, 1964.

[7] A. Spin, R. H Espinosa, et al., "Reactor nuclear IAN-R1: características técnicas y aplicaciones", Revista Técnico Científica del Instituto de Asuntos Nucleares, vol. 3, n. ${ }^{\text {os }} 5$ y 6, 1988, pp. 57-64.

[8] Lockheed Western Export Company, Propuesta reactor de investigaciones y entrenamiento avanzados para el Instituto de Asuntos Nucleares, Bogotá, en. 1964.

[9] J. M. González, "Modernización de la instrumentación de control y seguridad del reactor IAN-R1", Revista Técnico Científica del Instituto de Asuntos Nucleares, vol. 6, n. ${ }^{\text {os }} 11$ y 14,1992 , pp. 21-32.

[10] Presidencia de la República de Colombia, Decreto 2119 de 1992, "Por el cual se reestructura el Ministerio de Minas y Energía, el Instituto de Asuntos Nucleares (IAN) y Minerales de Colombia S. A.", Diario Oficial, n. ${ }^{\circ}$ 40.704, título IV, 1992.

[11] Presidencia de la República de Colombia, Decreto 1682 de 1997, "Por el cual se suprime el Instituto de Ciencias Nucleares y Energías Alternativas (INEA)", Diario Oficial, n. ${ }^{\circ} 43.072,1997$.

[12] International Atomic Energy Agency, "Government of the Republic of Colombia Represented by the Instituto de Ciencias Nucleares y Energías Alternativas, General Atomics. Upgrade of the INEA R1 Research Reactor at Santafé de Bogotá, Colombia”, Contract 1994-319.

[13] General Atomics, "Safety Analisys Report for the Research Reactor Conversion to TRIGA LEU Fuel at In- stituto de Ciencias Nucleares y Energías Alternativas", 1995.

[14] Presidencia de la República de Colombia, Decreto 1452 de 1998, "Por el cual se distribuyen unas funciones del Instituto de Ciencias Nucleares y Energías Alternativas (INEA), en liquidación, en el Instituto de Investigación en Geociencias, Minería y Química (Ingeominas)”. Diario Oficial, n. ${ }^{\circ}$ 43.353, Bogotá, 1998.

[15] Ingeominas, Libro de registro de operaciones reactor IAN-R1: Libro n. ${ }^{\circ}$ 10, Bogotá, 1991.

[16]Departamento Administrativo de Ciencia, Tecnología e Innovación, Centro Internacional de Física, Servicio Geológico Colombiano, "Convenio Especial de Cooperación n. ${ }^{\circ}$ 716-2009”, Bogotá, 2009.

[17] Centro Internacional de Física, Instituto Nacional de Investigaciones Nucleares, Contrato de Compraventa n. ${ }^{\circ}$ 01-2012 entre el Centro Internacional de Física (CIF), Instituto Nacional de Investigaciones Nucleares (ININ) para la Renovación de la Instrumentación y Control del reactor nuclear IAN-R1 (Colombia)”, Bogotá, 2012.

[18] Instituto Nacional de Investigaciones Nucleares, "Sistema de supervisión y control de la instrumentación del reactor nuclear IAN-R1 colombiano", (SRC-SSI), 2012.

[19] Instituto Nacional de Investigaciones Nucleares, "Sistema de protección (SP) del reactor nuclear IAN-R1 (Colombia)", 2012.

[20] Instituto Nacional de Investigaciones Nucleares, "Sistema de supervisión y control de la instrumentación del reactor nuclear IAN-R1 colombiano", (HMI), 2012.

[21] Instituto de Asuntos Nucleares, Instituto de Ciencias Nucleares, 30 años (1959-1989), Bogotá, 1989.

[22] Ministerio de Minas y Energía, Resolución n. ${ }^{\circ} 181757$ de 26 dic. 2005, "Por la cual se crea un grupo de trabajo en la Dirección de Energía”, Bogotá, 2005.

[23] Ministerio de Minas y Energía, Resolución n. 181475 del 12 de noviembre de 2004, "Por la cual se expide el reglamento sobre instalaciones nucleares y se establecen los requisitos para la obtención de licencias para su operación, parada prolongada, modificación y desmantelamiento", Bogotá, 2004.

[24] Servicio Geológico Colombiano, Manual de la calidad (NTC-ISO/IEC 17025), versión 2, código MN-PSG-003, 2017. 
Servicio Geológico Colombiano

[25] Servicio Geológico Colombiano, Resolución n. ${ }^{\circ} 154$ de 2014, "Por la cual se crea el Comité de Seguridad del Reactor de Investigación IAN-R1", Bogotá, 2014.

[26] Servicio Geológico Colombiano, Archivos SGC, Bogotá, 2017.
[27] Instituto de Asuntos Nucleares, Libro de registro de operaciones reactor IAN-R1: Libro $n .{ }^{\circ}$ 1, Bogotá, 1965. 